\title{
Erratum to: Activation of Glycogen Synthase Kinase-3 Mediates the Olfactory Deficit-Induced Hippocampal Impairments
}

\author{
Juan Hu • He-Zhou Huang • Xiang Wang • Ao-Ji Xie • \\ Xiong Wang • Dan Liu $\cdot$ Jian-Zhi Wang • \\ Ling-Qiang Zhu
}

Published online: 13 January 2015

(C) Springer Science+Business Media New York 2015

\section{Erratum to: Mol Neurobiol}

DOI 10.1007/s12035-014-8953-9

The original paper of this article unfortunately contains error.

The correct information for the authorship should be "Juan Hu, He-Zhou Huang, Xiang Wang, Ao-Ji Xie, Xiong Wang, Dan Liu, Ling-Qiang Zhu, Jian-Zhi Wang, Juan Hu, He-Zhou Huang and Xiang Wang contributed equally to this paper."

The online version of the original article can be found at http://dx.doi.org/ 10.1007/s12035-014-8953-9.

J. Hu $\cdot$ H.-Z. Huang $\cdot$ X. Wang $\cdot$ A.-J. Xie $\cdot$ X. Wang $\cdot$ D. Liu $\cdot$ J.-Z. Wang $(\bowtie) \cdot$ L.-Q. Zhu $(\bowtie)$

Pathophysiology Department, Key Laboratory of Neurological

Disease of Education Committee of China, China-Canada

Cooperation Platform on Neurological Disorder, Institute of Brain

Research, School of Basic Medicine, Tongji Medical College,

Huazhong University of Science and Technology, Wuhan, People's

Republic of China

e-mail: wangjz@mails.tjmu.edu.cn

e-mail: zhulq@mail.hust.edu.cn

Present Address:

J. $\mathrm{Hu}$

Department of Oncology, The First College of Clinical Medical

Science, China Three Gorges University and Yichang Central

People's Hospital, No. 183 Yiling Road, Yichang, Hubei 443003,

China 